\title{
RESEARCH
}

\section{COVID-19 diagnosis from chest x-rays: developing a simple, fast, and accurate neural network}

\author{
Vasilis Nikolaou ${ }^{1 *} \mathbb{0}$, Sebastiano Massaro ${ }^{1,2}$, Masoud Fakhimi ${ }^{1}$, Lampros Stergioulas ${ }^{3}$ and Wolfgang Garn ${ }^{1}$
}

\begin{abstract}
Purpose: Chest $x$-rays are a fast and inexpensive test that may potentially diagnose COVID-19, the disease caused by the novel coronavirus. However, chest imaging is not a first-line test for COVID-19 due to low diagnostic accuracy and confounding with other viral pneumonias. Recent research using deep learning may help overcome this issue as convolutional neural networks (CNNs) have demonstrated high accuracy of COVID-19 diagnosis at an early stage.

Methods: We used the COVID-19 Radiography database [36], which contains x-ray images of COVID-19, other viral pneumonia, and normal lungs. We developed a CNN in which we added a dense layer on top of a pre-trained baseline CNN (EfficientNetB0), and we trained, validated, and tested the model on 15,153 X-ray images. We used data augmentation to avoid overfitting and address class imbalance; we used fine-tuning to improve the model's performance. From the external test dataset, we calculated the model's accuracy, sensitivity, specificity, positive predictive value, negative predictive value, and F1-score.
\end{abstract}

Results: Our model differentiated COVID-19 from normal lungs with 95\% accuracy, 90\% sensitivity, and 97\% specificity; it differentiated COVID-19 from other viral pneumonia and normal lungs with 93\% accuracy, 94\% sensitivity, and $95 \%$ specificity.

Conclusions: Our parsimonious CNN shows that it is possible to differentiate COVID-19 from other viral pneumonia and normal lungs on $\mathrm{x}$-ray images with high accuracy. Our method may assist clinicians with making more accurate diagnostic decisions and support chest X-rays as a valuable screening tool for the early, rapid diagnosis of COVID-19.

Keywords: COVID-19, Chest x-rays, Artificial intelligence, Deep learning, Classification, Convolution Neural Network

\section{Introduction}

COVID-19 is an infectious disease caused by severe acute respiratory syndrome coronavirus 2 (SARS-CoV-2) [1]. The virus was first observed in Wuhan, China, in December 2019 and then spread globally. On March 11, 2020, the World Health Organization (WHO) declared COVID-19 a pandemic [1]. The virus spreads via respiratory droplets or aerosol so that it can be transmitted into individuals' mouth, nose, or eyes of individuals in close

\footnotetext{
*Correspondence: v.nikolaou@surrey.ac.uk

${ }^{1}$ Surrey Business School, University of Surrey, Alexander Fleming Rd, Guildford GU2 7XH, UK

Full list of author information is available at the end of the article
}

proximity. The most common symptoms include a high fever, continuous cough, and breathlessness [2].

COVID-19 is usually diagnosed by an RT-PCR test [3] and often is complemented by chest radiographs, including $\mathrm{x}$-ray images and computed tomography (CT) scans [4]. X-ray machines are widely available worldwide and provide images quickly, so chest scans have been recommended, by some researchers [5], for screening during the pandemic. Unlike the RT-PCR test, chest scans provide information about both the status of infection (i.e., presence or absence of the disease) and disease severity. Moreover, $\mathrm{x}$-ray imaging is an efficient and cost-effective procedure. It requires relatively cheap equipment and can be performed rapidly in isolated rooms with a portable 
chest radiograph (CXR) device, thus reducing the risk of infection inside hospitals $[6,7]$.

Despite these benefits, the American College of Radiology (ACR) and the Centers for Disease Control and Prevention $(\mathrm{CDC})$ have not endorsed chest imaging as a first-line test for COVID-19 [8]. In the diagnosis of COVID-19, chest CT scans are highly sensitive (97\%) but far less specific (25\%) than RT-PCR [9]. Recent research, however, suggests that deep learning techniques may improve the specificity of $x$-ray imaging [10] for the diagnosis of COVID-19 (see publications in Table 1).

Many of the studies in Table 1 use convolutional neural networks (CNNs) that take advantage of transfer learning-that is, they are built on existing CNNs (e.g., EfficientNet, VGG-16, AlexNet, GoogLeNet, SqueezeNet, ResNet) that were trained on large-scale image-classification datasets (e.g., ImageNet [11]). The size of the dataset and generic nature of the trained task (e.g., describe the general shapes of an object) make the features learned by the existing $\mathrm{CNN}$ useful for other computer-vision problems with other images. As shown in Table 1, most of the studies reported high accuracy (above 87\%) [12-32], ten studies reported high sensitivity (above $88 \%$ ) [12, 13, 16$18,29,30,33-35]$, seven studies reported high specificity (above 78\%) [14, 15, 17, 18, 28, 29, 31, 34, 35], three studies reported high F1-scores (above 94\%) [14, 15, 30], and three studies reported above $94 \%$ precision [12-14]. All of the models were validated internally (i.e., trained and validated on different random splits of the same dataset; the proportions of the dataset for training versus validation vary). However, it remains unclear whether these models would perform as well on an external validation task (i.e., on a different dataset that was not involved in the model's development).

To address the need for external validation and advance the possibility of using $x$-ray technology to alleviate the impact of the global pandemic, we develop a CNN using a parsimonious yet powerful pre-trained $\mathrm{CNN}$ as the baseline model, and we assess its diagnostic accuracy on an independent (external) dataset. We compare the model's performance on two classification datasets: a) COVID-19 vs. normal lungs (two-class classification) and b) COVID-19 vs. other viral pneumonia vs. normal lungs (three-class classification).

\section{Methods}

\section{Study design}

We used the COVID-19 Radiography database [36], a public database that contains 15,153 images (as of 3rd May 2021) across three cohorts: COVID-19, other viral pneumonia, and normal lungs. We randomly split each of the three cohorts into train (70\%), validation (20\%), and test (10\%) subsets (Fig. 1). Only the train and validation subsets contributed to the model's development; we kept the test subset separate for external validation.

\section{Transfer learning and feature extraction}

We developed a hybrid CNN using a pre-trained ConvNet called EfficientNetB0 [37], which is the baseline model of a family of EfficientNet models (from EfficientNetB0 to EfficientNetB7). These models use compound scaling, in which the image's dimensions (i.e., depth, width, and resolution) are scaled by a fixed amount at the same time. Models with compound scaling usually outperform other CNNs, as shown in Fig. 2: the baseline B0 model starts at a higher accuracy than some other models (e.g., ResNet-50, Inception-v2), while the latest EfficientNet model (B7) achieves the highest accuracy of all (84\%). Although the EfficientNets achieve high accuracy, they require fewer parameters (5 million for B0; 66 million for B7) and less computation time than most other models. In this work, we utilize the EfficientNet-B0 as our baseline model for the following reasons: (a) it has less parameters than the rest models (B1-B7) of the EfficientNet family, (b) it is more cost-efficient for training and tuning than the more advanced EfficientNetB1-B7 model as it does not require much computational power (see Discussion section) and (c) it contributes to high accuracy in differentiating COVID-19 from non-COVID-19 viral pneumonia and healthy images (see Results section), thus satisfies the rational of our study for developing a parsimonious yet powerful convolution network.

On top of the baseline EfficientNetB0 network, we connected a fully dense layer of 32 neurons (Fig. 3). The features learned by the baseline model are run through our (two-class or three-class) classifier to extract new features from the sample.

Our CNN uses about 5 million parameters (Fig. 4), which is considerably fewer than AlexNet (61 million) and GoogLeNet (7 million) [14]. Thus, our CNN was faster to train and less likely to overfit the training data, which leads to worse performance on external datasets. We also reduced the risk of overfitting by adding $20 \%$ and $50 \%$ (which outperformed the 20\% drop-out rate for the threeclass model; results not shown) drop-out rates to our twoclass and three-class prediction models, respectively. By adding drop-out during training, a proportion of features (20\% and $50 \%$ for the two- and three-class model respectively) is set to zero, whereas during validation, all features 


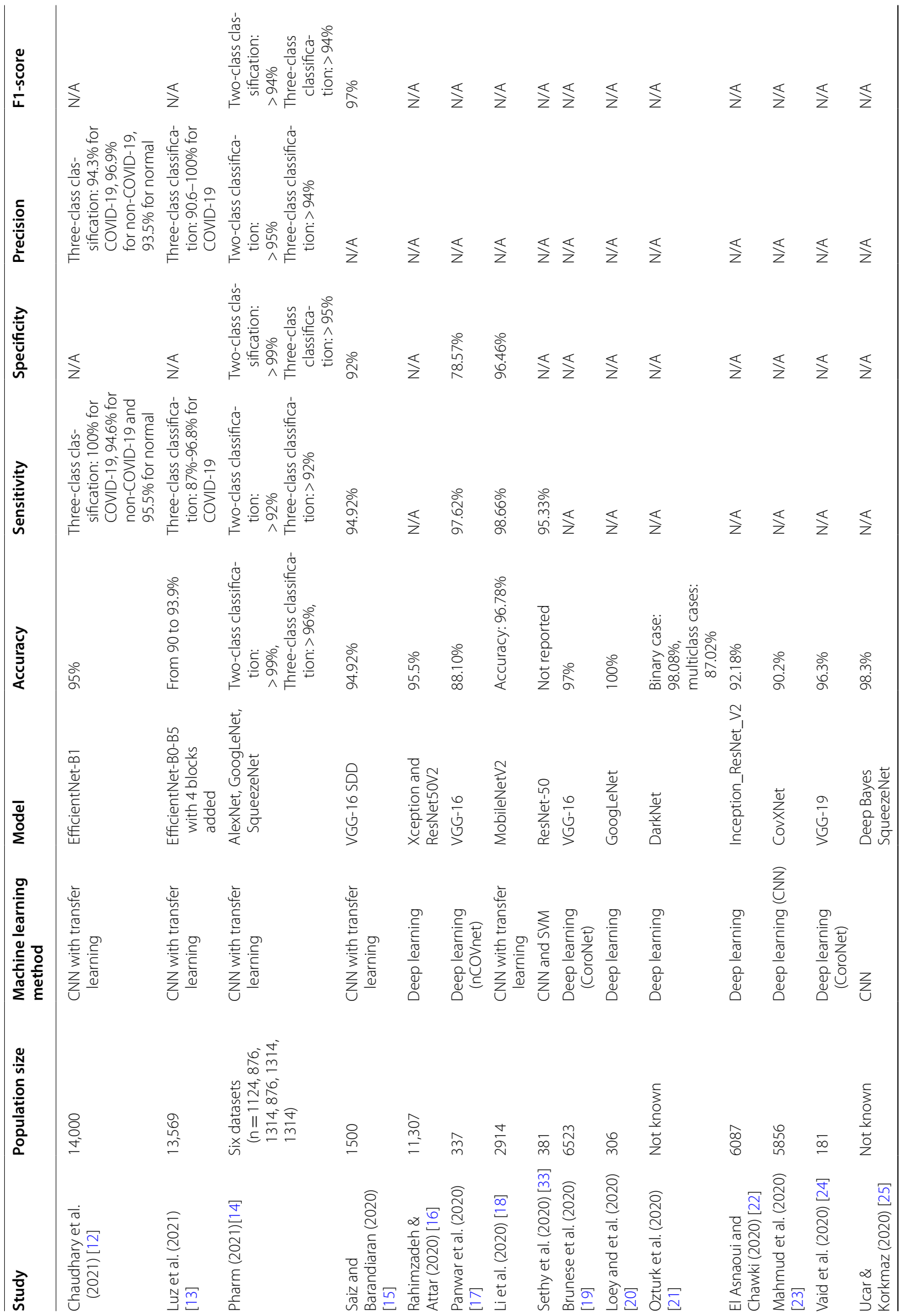




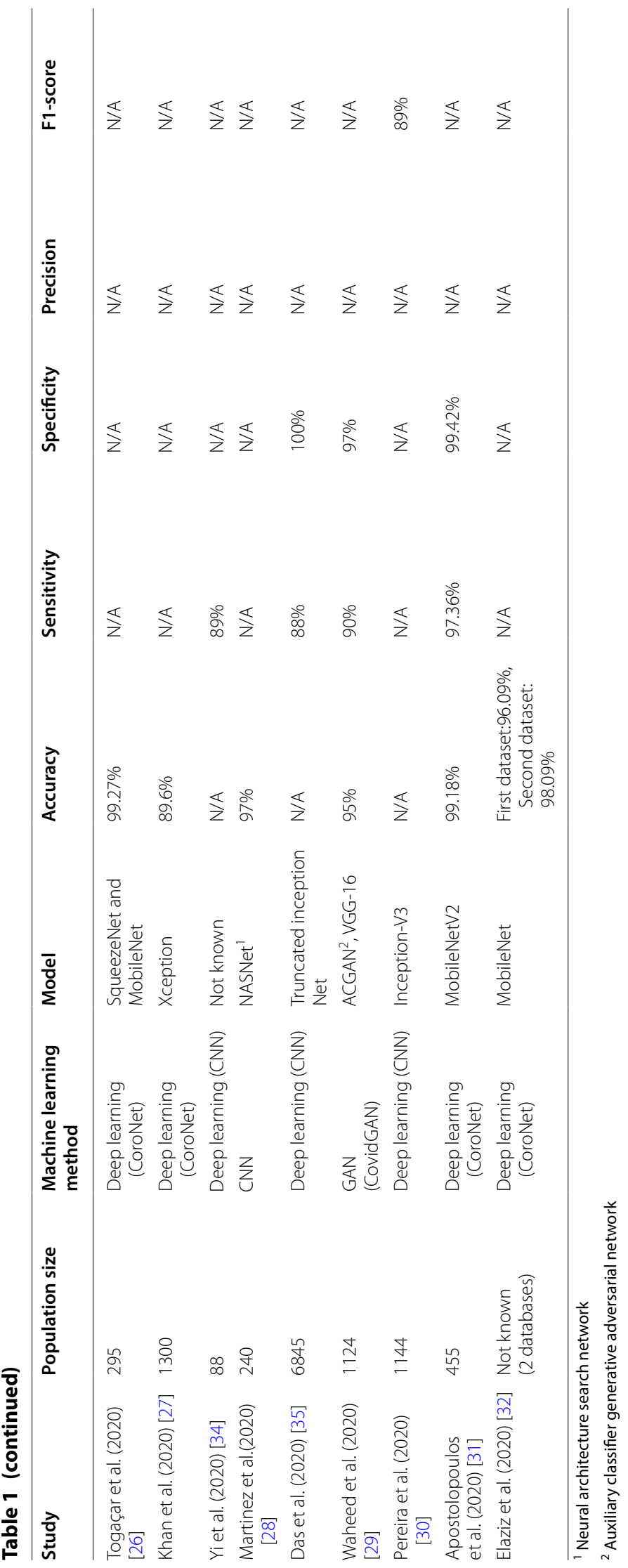




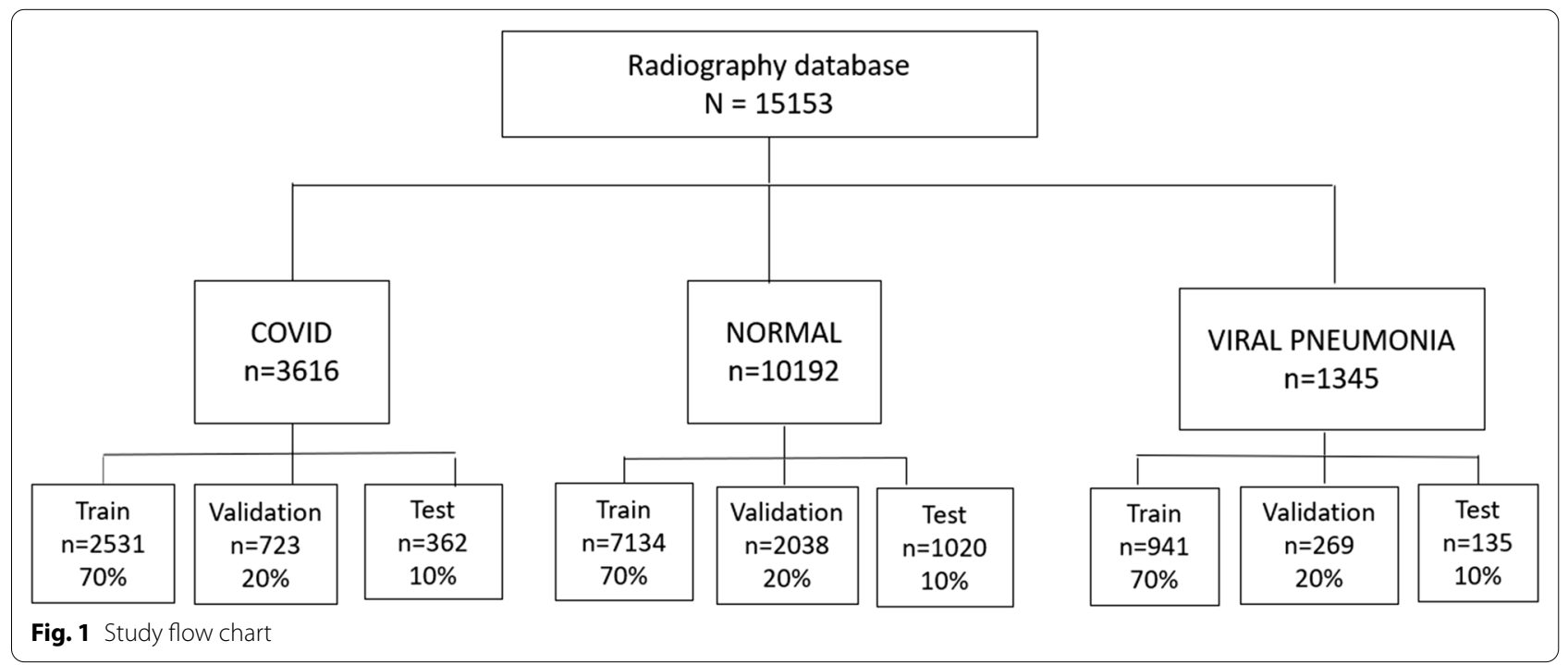

are used. This makes the model at validation more robust, leading to higher testing accuracy (Figs. 5 and 6). We applied Sigmoid and Softmax operations to model the two- and three-class classification outputs, respectively. We used an Adam optimizer with a learning rate of 0.001 .

Before training the model, we "froze" the convolution base (i.e., EfficientNetB0) to preserve the representations learned during the baseline model's original training. Subsequently, we trained only the weights in the two dense layers that we added to the convolution base.

\section{Data augmentation}

We augmented the training data to eliminate the class size imbalance and avoid overfitting. First, we resized the images to $150 \times 150$ pixels to reduce the data volume, and we normalized them to the $[0,1]$ interval because neural networks are more efficient with small input values. Then, we augmented the training images through random transformations to increase the variety of the images. Specifically, we manipulated the parameters by (a) rotating the image by 40 degrees, (b) randomly shifted the height and width horizontally or vertically by $20 \%$ of the image's initial size, (c) randomly clipping the image by $20 \%$, (d) randomly zooming in by $20 \%$, (e) randomly flipping half of the images horizontally, and (f) filling in the pixels that were created by a rotation or a height or width shift. Data augmentation is essential for avoiding overfitting in small samples because the additional, varied images prevent the $\mathrm{CNN}$ from being exposed to the same image twice.

\section{Fine-tuning}

We fine-tuned our CNN by "unfreezing" a few of the top layers (i.e., from block $7 \mathrm{a}$ onwards) in the convolution base and jointly training both the "unfrozen" layers and the two layers that we added (Fig. 7). By training some of the top layers of the baseline $\mathrm{CNN}$, we adjusted the presentations of the pre-trained model that were more abstract (in terms of shape and size) to make them more relevant and specific to our sample, thereby improving our model's performance.

\section{Performance metrics}

We assessed the model's performance with the following metrics [14]:

(a) sensitivity (or recall): the percentage of positive cases that were predicted to be positive

$$
\text { Sensitivity }=\frac{\text { True Positives }}{\text { True Positives }+ \text { False Negatives }}
$$

(b) specificity: the percentage of negative cases that were predicted to be negative

$$
\text { Specificity }=\frac{\text { True Negatives }}{\text { True negatives }+ \text { False positives }}
$$




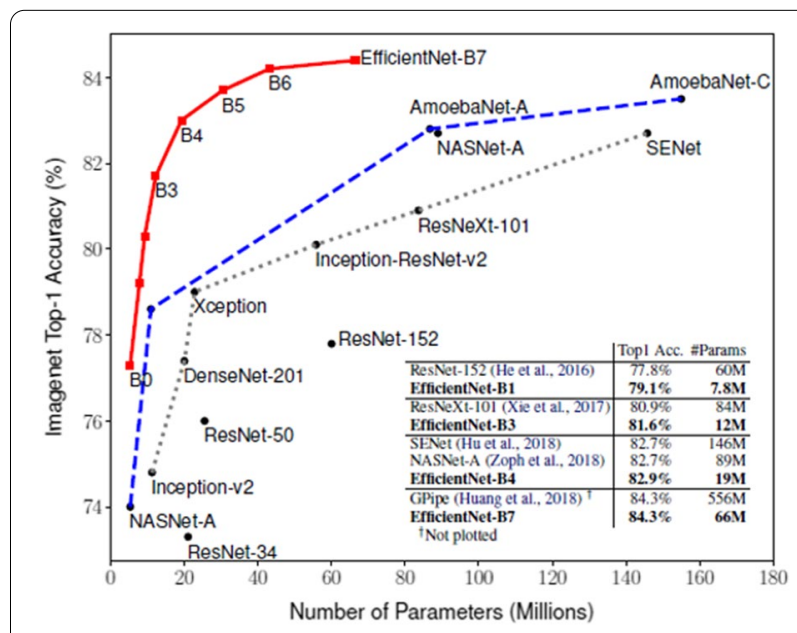

Fig. 2 The performance of the EfficientNet models versus other CNNs on ImageNet (from Tan \& Lee 2019). Source: Tan M, Le Q, Efficientnet: Rethinking model scalling for convolutional neural networks. In International Conference on Machine Learing 2019 May 24 (pp. 6105-6114). PMLR

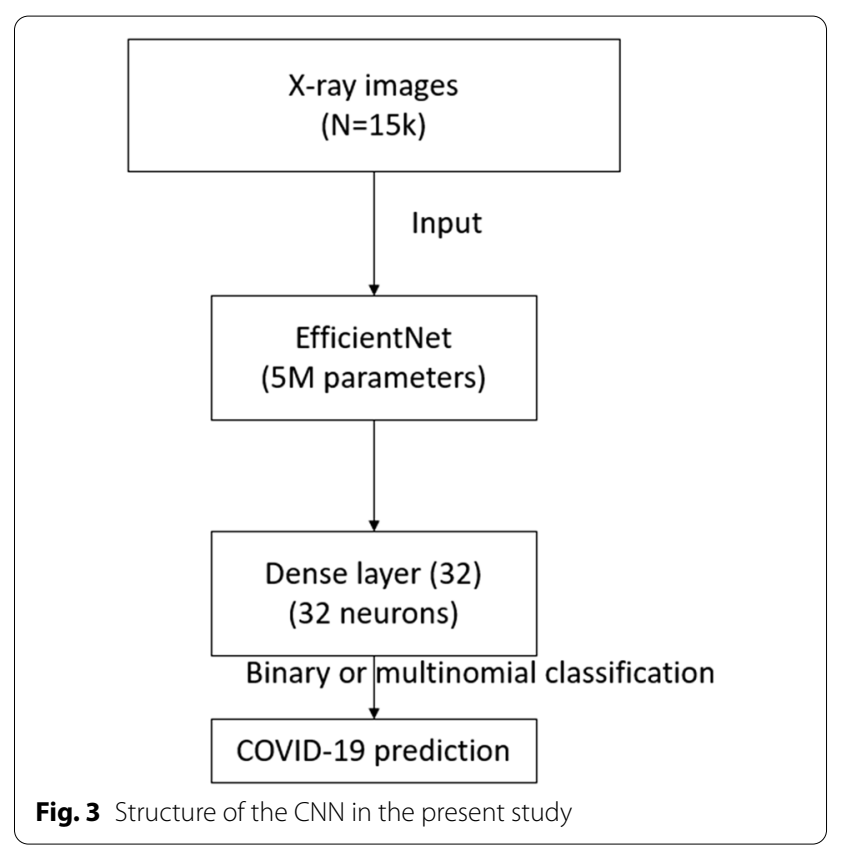

(c) positive predictive value (or precision): the percentage of positive predictions that were actually positive cases

$$
\text { Positive predictive value }=\frac{\text { True Positives }}{\text { True Positives }+ \text { False Positives }}
$$

(d) negative predictive value: the percentage of negative predictions that were actually negative cases (e) F1-score: a combination of recall and precision, used for model comparison

$$
F 1-\text { score }=\frac{2 X \text { True Positives }}{2 X \text { True Positives }+ \text { False Positives }+ \text { False Negatives }}
$$

(f) accuracy

$$
\text { Accuracy }=\frac{\text { True Positives }+ \text { True Negatives }}{\text { Total number of cases }}
$$

Moreover, we constructed 95\% confidence intervals for the above metrics using bootstrapping.

\section{Results}

We include results for the two-class classification (COVID-19 vs. normal lungs) and the three-class classification (COVID-19 vs. other viral pneumonia vs. normal lungs).

\section{Two-class classification}

Table 2 reports the performance of the two-class classification model with feature extraction only and with fine-tuning. Figure 5 depicts the performance after finetuning on the train (blue line) and validation subsets (orange line). The model reached an accuracy of $93.8 \%$ on the validation subset after training for 10 epochs (i.e., runs through the train dataset).

As shown in Table 2, the model's performance improved with fine-tuning, achieving a recall (i.e., sensitivity) of $90 \%$ (95\% CI 88, 92), specificity of 97\% (95\% CI 96, 98), precision (i.e., PPV) of 91\% (95\% CI 89, 93), F1-score of $90 \%(95 \%$ CI 88, 92), and accuracy of $95 \%$ (95\% CI 94, 96). The fine-tuned model misclassified only 32 normal images as COVID-19 (versus 70 misclassifications in the model with only feature extraction).

\section{Three-class classification}

Table 3 reports the performance of the three-class classification model with feature extraction only and with fine-tuning. Figure 6 depicts the performance after finetuning on the train (blue line) and validation subsets (orange line). The model reached an accuracy of $92.6 \%$ after 10 epochs.

As shown in Table 3, the model's performance improved with fine-tuning, achieving an accuracy of $93 \%$ (95\% CI 92, 95), recall (i.e., sensitivity) of 94\% (95\% CI 93, 96), precision (i.e., PPV) of $86 \%$ (95\% CI 84, 88), and F1-score of $90 \%(95 \%$ CI 88, 92). The F1-score improved

$$
\text { Negative predictive value }=\frac{\text { True Negatives }}{\text { True Negatives }+ \text { False Negatives }}
$$




\begin{tabular}{|c|c|c|}
\hline 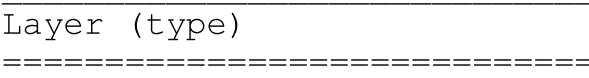 & $\begin{array}{l}\text { Output Shape } \\
================\end{array}$ & $\begin{array}{l}\text { Parameters } \\
=========\end{array}$ \\
\hline efficientnet-b0 (Functional) & (None, 5, 5, 1280) & 4049564 \\
\hline flatten_1 (Flatten) & (None, 32000$)$ & 0 \\
\hline dense_2 (Dense) & (None, 32) & 1024032 \\
\hline dropout_1 (Dropout) & (None, 32) & 0 \\
\hline dense_3 (Dense) & (None, 2) & 66 \\
\hline$=0=$ & $=============$ & $====$ \\
\hline $\begin{array}{l}\text { Total params: } 5,073,662 \\
\text { Trainable params: 5,031,646 } \\
\text { Non-trainable params: } 42,016\end{array}$ & & \\
\hline
\end{tabular}

Fig. 4 Summary of the parameters for the two-class classification

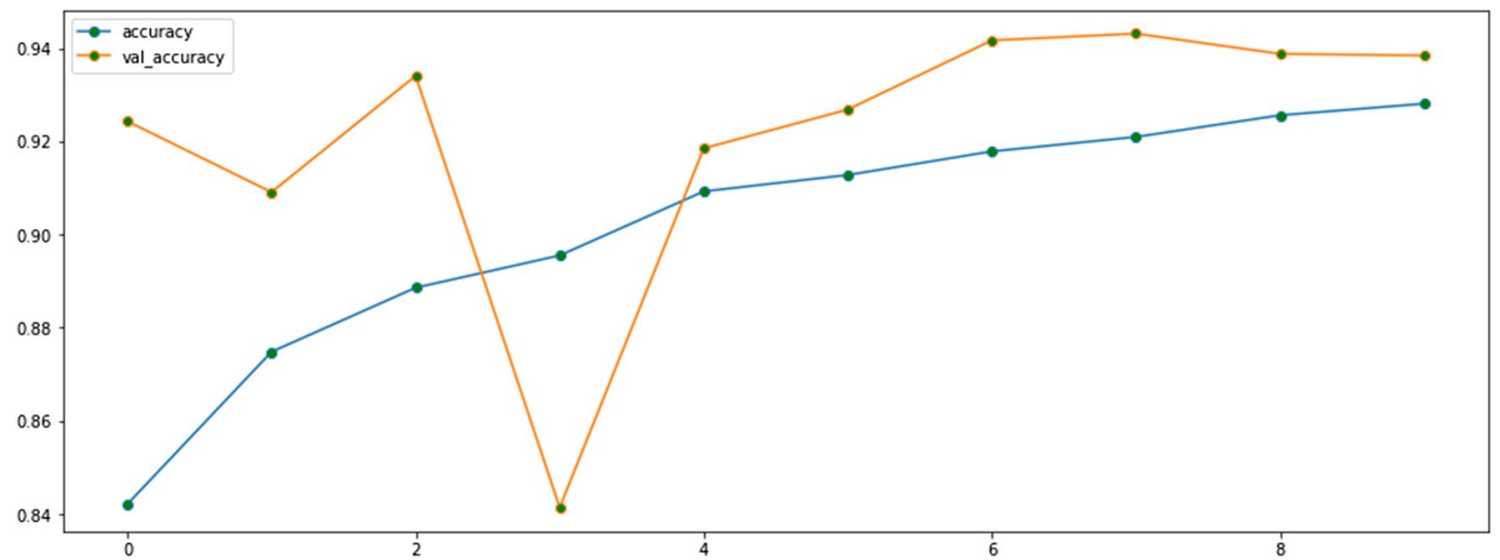

Fig. 5 The fine-tuned model's performance on the train and validation subsets for two-class classification

by $5 \%$ with fine-tuning relative to the model with only feature extraction. The fine-tuned model was adept at differentiating between COVID-19 and other viral pneumonia-only one other viral pneumonia image was misclassified as COVID-19, and no COVID-19 images were misclassified as other viral pneumonia. (Two normal images were misclassified as other viral pneumonia.).

\section{Discussion}

We implemented a hybrid $\mathrm{CNN}$ that combines a pretrained EfficientNetB0 network with a dense layer (32 neurons) to differentiate between $x$-ray images of COVID-19 and normal lungs (and other viral pneumonia, in the three-class classification). After feature extraction and fine-tuning, the model achieved 95\% (95\% CI 94, 96) accuracy for the two-class classification and 93\% (95\% CI 92, 95) accuracy for the three-class classification. This model's performance is comparable to existing models $[10,12,13]$, but it offers several other advantages.

Methodologically, to the best of our knowledge, this is the first instance in which the pre-trained EfficientNetB0 (the baseline model of the EfficientNet family, which uses compound scaling to achieve higher accuracy) with a dense layer(32) on top has been used to improve the accuracy of COVID-19 diagnosis from X-ray images. Chaudhary et al. [12] used an EfficientNet-B1 model to 


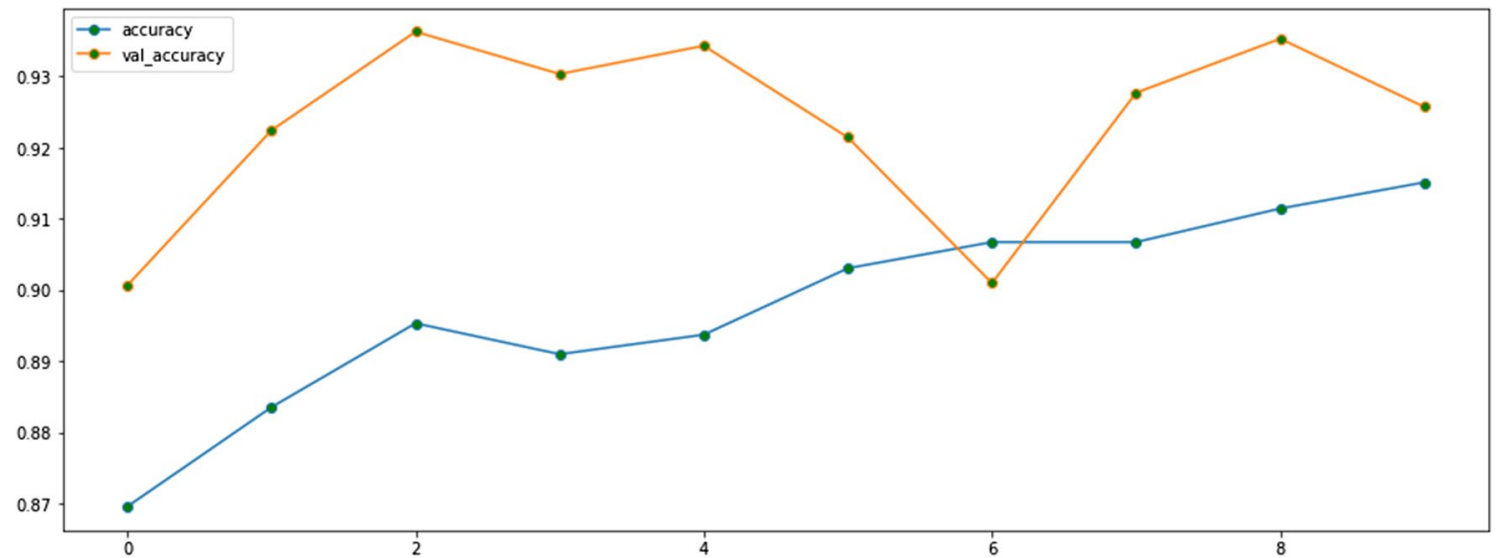

Fig. 6 The fine-tuned model's performance on the train and validation subsets for three-class classification

distinguish COVID-19 from non-COVID-19 and normal x-ray images with 95\% accuracy and 100\% sensitivity, while Luz et al. [13] used a range of EficientNetB0-B5 models with four dense layers on top. In the latter case, the EfficientNet-B0 with four layers on top achieved an accuracy of $90 \%$ and sensitivity of $93.5 \%$, whereas the best performing EfficientNet-B3 with four layers on top achieved 94\% accuracy and $96.8 \%$ sensitivity. In comparison, our model achieved better accuracy (93\%) and slightly better sensitivity (94\%) than the B0-X (where X denotes the four layers on top) with fewer parameters (5 million vs 5.3 million). It also achieved similar accuracy and sensitivity with the $\mathrm{B} 1$ and $\mathrm{B} 3-\mathrm{X}$ (6.6 and 12.3 million parameters respectively). Jiao et al. [38] also used EfficientNet as the baseline

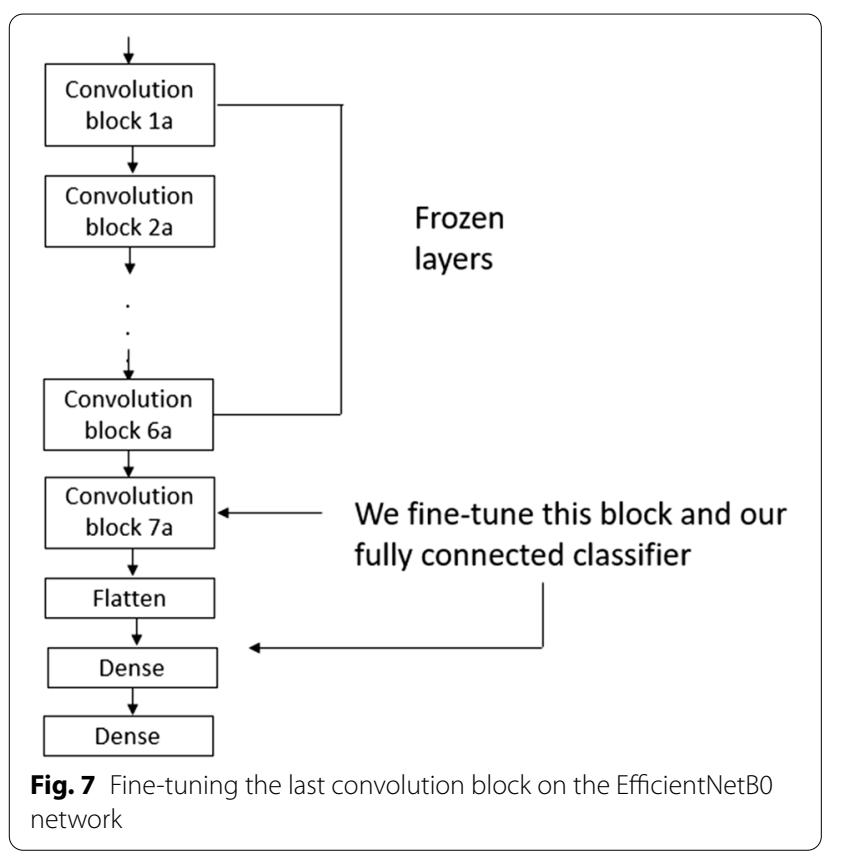

ConvNet, but the prediction was different (COVID-19 severity: critical vs. non-critical), and the model was considerably more complex: it connected a convolutional layer (256 neurons) and a dense layer (32 neurons) on top of the baseline. Despite its complexity, the model did not perform as well on an external dataset: accuracy of $75 \%$ (95\% CI 74, 77), sensitivity of $66 \%(95 \%$ CI 64,68$)$, and

Table 2 The model's performance on two-class classification

\begin{tabular}{|c|c|c|}
\hline \multirow[t]{2}{*}{ Model with feature extraction } & \multicolumn{2}{|l|}{ Predicted } \\
\hline & Normal & COVID-19 \\
\hline \multicolumn{3}{|l|}{ Observed } \\
\hline Normal & 950 & 70 \\
\hline COVID-19 & 47 & 315 \\
\hline Accuracy (95\% Cl) (\%) & $92(90,94)$ & \\
\hline Sensitivity (95\% CI) (\%) & $87(85,89)$ & \\
\hline Specificity (95\% CI) (\%) & $93(91,95)$ & \\
\hline PPV (95\% CI) (\%) & $82(79,84)$ & \\
\hline NPV (95\% Cl) (\%) & $95(94,96)$ & \\
\hline F1-score $(95 \%$ Cl) (\%) & $84(82,86)$ & \\
\hline \multirow[t]{2}{*}{ Model with fine-tuning } & \multicolumn{2}{|l|}{ Predicted } \\
\hline & Normal & COVID-19 \\
\hline \multicolumn{3}{|l|}{ Observed } \\
\hline Normal & 988 & 32 \\
\hline COVID-19 & 38 & 324 \\
\hline Accuracy (95\% Cl) (\%) & $95(94,96)$ & \\
\hline Sensitivity (95\% Cl) (\%) & $90(88,92)$ & \\
\hline Specificity (95\% Cl) (\%) & $97(96,98)$ & \\
\hline PPV (95\% CI) (\%) & $91(89,93)$ & \\
\hline NPV (95\% Cl) (\%) & $96(95,97)$ & \\
\hline F1-score $(95 \%$ Cl) (\%) & $90(88,92)$ & \\
\hline
\end{tabular}

$\mathrm{Cl}$ confidence interval, $P P V$ positive predictive value, $N P V$ negative predictive value 
specificity of $70 \%$ (95\% CI 69, 71). Although the present model addresses a different problem, we are confident that it performs better on the two-class classification problem than the model built by Jiao et al. Given that a model trained on more parameters is more prone to overfitting, we believe that our relatively small number of parameters and data augmentation strategy contributed to our model's superior performance.

Another advantage of this study lies in its design. Previous works have used a variety of splits to create subsets of data for two purposes: training and validation. For instance, Pham [14] used several random splits $(80 \%$ vs. $20 \% ; 50 \%$ vs. $50 \%$; $90 \%$ vs. $10 \%$ ) of an older version of the COVID-19 Radiography database used here. Rahimzadeh et al. [16] used eight subsets of their dataset for training and another subset for testing; Panwar et al. [17] used $70 \%$ for training and $30 \%$ for validation. To the best of our knowledge, the present research is the first to cluster the dataset into three independent subsets: train (70\%), validation $(20 \%)$, and test (10\%). Thus, we reduced overfitting by testing the model's performance on a subset of data that did not contribute to the model's development.
We also mitigated overfitting by augmenting the training images (i.e., randomly transforming existing images to generate new ones). Data augmentation also addressed the class imbalance (as there were unequal numbers of COVID-19, other viral pneumonia, and normal images). We fine-tuned the model by training some of the top layers of our baseline $\mathrm{CNN}$ to improve their specificity to the current problem.

Finally, all of the reviewed studies except for one [14] reported only point estimates for their performance metrics (accuracy, sensitivity, specificity, positive predictive value, negative predictive value, and F1-score). By including the $95 \%$ confidence intervals, we capture the uncertainty of our estimates and enable a more comprehensive appraisal of the model.

The main limitation of our study is the lack of patient data. We appreciate that the model built by Jiao et al. [38] included patient clinical data (e.g., age, sex, oxygen saturation, biomarkers, comorbidities), which slightly improved the accuracy of the image-based $\mathrm{CNN}$. We recognize that this is an important avenue for future research. We further note that the training and tuning of

Table 3 The model's performance on three-class classification

\begin{tabular}{|c|c|c|c|}
\hline \multirow[t]{2}{*}{ Model with feature extraction } & \multicolumn{3}{|l|}{ Predicted } \\
\hline & Normal & Other viral pneumonia & COVID-19 \\
\hline \multicolumn{4}{|l|}{ Observed } \\
\hline Normal & 941 & 0 & 79 \\
\hline Other viral pneumonia & 20 & 108 & 7 \\
\hline COVID-19 & 32 & 0 & 330 \\
\hline Accuracy $(95 \%$ Cl) (\%) & $91(89,93)$ & & \\
\hline Sensitivity (95\% Cl) (\%) & $91(89,93)$ & & \\
\hline Specificity (95\% CI) (\%) & $92(90,94)$ & & \\
\hline PPV (95\% Cl) (\%) & $79(77,82)$ & & \\
\hline NPV (95\% Cl) (\%) & $95(94,96)$ & & \\
\hline F1-score $(95 \%$ Cl) $(\%)$ & $85(83,87)$ & & \\
\hline \multirow[t]{2}{*}{ Model with fine-tuning } & \multicolumn{3}{|l|}{ Predicted } \\
\hline & Normal & Other viral pneumonia & COVID-19 \\
\hline \multicolumn{4}{|l|}{ Observed } \\
\hline Normal & 964 & 2 & 54 \\
\hline Other viral pneumonia & 32 & 102 & 1 \\
\hline COVID-19 & 22 & 0 & 340 \\
\hline Accuracy $(95 \%$ Cl) (\%) & $93(92,95)$ & & \\
\hline Sensitivity (95\% Cl) (\%) & $94(93,96)$ & & \\
\hline Specificity (95\% CI) (\%) & $95(94,96)$ & & \\
\hline PPV (95\% Cl) (\%) & $86(84,88)$ & & \\
\hline NPV (95\% CI) (\%) & $95(94,96)$ & & \\
\hline F1-score (95\% CI) (\%) & $90(88,92)$ & & \\
\hline
\end{tabular}

$\mathrm{Cl}$ confidence interval, $P P V$ positive predictive value, $N P V$ negative predictive value 
our "light" CNN took about two hours on a conventional Macintosh computer with 16G RAM and one Terabyte of a hard disk. More computational power is instead needed to train more advanced versions of the EfficientNet (from B1 through B7), possibly increasing the barrier of entry for non-specialists users (i.e., clinicians).

\section{Conclusions}

This study uses a "light" CNN to discriminate COVID-19 from other viral pneumonia and healthy lungs in chest $\mathrm{X}$-ray images. To the best of our knowledge, our model is at present the most parsimonious $\mathrm{CNN}$ used to address the demanding issue of COVID-19 diagnosis via chest $\mathrm{x}$-ray. The model successfully overcame the issue of low specificity that has prevented the ACR and CDC from endorsing chest imaging to diagnose COVID-19 [8]. Specifically, the fine-tuned model differentiated COVID19 from normal lungs with a positive predictive value of $91 \%$ and specificity of $97 \%$ (95\% CI 96, 98); it differentiated COVID-19 from other viral pneumonia and normal lungs with a positive predictive value of $86 \%$ and specificity of $95 \%(95 \%$ CI 94, 96). Both classifications had a negative predictive value of $95 \%$ (95\% CI 94, 96), meaning that a negative COVID-19 classification would indicate a 95\% chance of not having COVID-19. Moreover, as shown in Table 3, just one image of other viral pneumonia was misclassified as COVID-19, proving a remarkable discriminatory ability given the overlap in the presentation of COVID-19 and other viral cases of pneumonia.

The insights presented in this study may help clinicians (namely, radiologists) accurately diagnose COVID-19 at an early stage by enabling the use of $\mathrm{x}$-ray as a first-totest tool that can complement RT-PCR analyses. Moving forward, the validation of our $\mathrm{CNN}$ on other databases would increase our confidence in the use of neural networks to aid the early diagnosis of both COVID-19 and other life-threatening diseases that traditionally have been difficult to diagnose via imaging.

\section{Supplementary Information}

The online version contains supplementary material available at https://doi. org/10.1007/s13755-021-00166-4.

Below is the link to the electronic supplementary material.Supplementary file 1 (DOCX $40 \mathrm{~kb}$ )

\section{Funding}

The author received no funding for this work.

\section{Data Availability}

The publicly available COVID-19 Radiography Database [36] was used.

\section{Code availability}

The python notebook for the two-class classification model is available here: http://localhost:8888/notebooks/OneDrive\%20-\%20University\%20of\%20 Surrey/Kaggla_data/Two-class_classification.ipynb. The python notebook for the three-class classification model is available here: http://localhost:8888/ notebooks/OneDrive\%20-\%20University\%20of\%20Surrey/Kaggla_data/Threeclass_classification.ipynb.

\section{Declarations}

Conflict of interest

The author declares no conflict of interest.

\section{Author details}

${ }^{1}$ Surrey Business School, University of Surrey, Alexander Fleming Rd, Guildford GU2 7XH, UK. ${ }^{2}$ The Organizational Neuroscience Laboratory, London WC1N 3AX, UK. ${ }^{3}$ The Hague University of Applied Sciences, Johanna Westerdijkplein 75, 2521 EN Den Haag, The Netherlands.

Received: 15 June 2021 Accepted: 20 September 2021

Published online: 12 October 2021

\section{References}

1. COVID-19 pandemic. https://en.wikipedia.org/wiki/COVID-19_pandemic. Accessed on 09 May, 2021

2. COVID-19 pandemic. https://en.wikipedia.org/wiki/COVID-19_pande mic\#Transmission. Accessed on 09 May, 2021

3. Reverse transcription polymerase chain reaction. https://en.wikipedia. org/wiki/Reverse_transcription_polymerase_chain_reaction. Accessed on 09 May, 2021

4. COVID-19 pandemic. https://en.wikipedia.org/wiki/COVID-19_pande mic\#Diagnosis. Accessed on 09 May, 2021

5. Ng M-Y, Lee EYP, Yang J, et al. Imaging profile of the COVID-19 infection: radiologic findings and literature review. Radiology. 2020;2(1):e200034.

6. Baratella E, Crivelli P, Marrocchio C, et al. Severity of lung involvement on chest $\mathrm{X}$-rays in SARS-coronavirus-2 infected patients as a possible tool to predict clinical progression: an observational retrospective analysis of the relationship between radiological, clinical, and laboratory data. J Brasil Pneumol. 2020;46(5):e20200226.

7. Rubin GD, Ryerson CJ, Haramati LB, et al. The role of chest imaging in patient management during the COVID-19 pandemic: a multinational consensus statement from the Fleischner society. Chest. 2020;296(1):172-80.

8. ACR Recommendations for the use of Chest Radiography and Computed Tomography (CT) for Suspected COVID-19 Infection (https://www.acr. org/Advocacy-and-Economics/ACR-Position-Statements/Recommenda tions-for-Chest-Radiography-and-CT-for-Suspected-COVID19-Infection). Accessed on 09/05/2021.

9. Ai T, Yang Z, Hou H, Zhan C, Chen C, Lv W, Tao Q, Sun Z, Xia L. Correlation of chest CT and RT-PCR testing for coronavirus disease 2019 (COVID-19) in China: a report of 1014 cases. Radiology. 2020;296(2):E32-40.

10. Ghaderzadeh M, Asadi F. Deep learning in the detection and diagnosis of COVID-19 using radiology modalities: a systematic review. J Healthcare Eng. 2021;15:2021.

11. Deng J, Dong W, Socher R, Li L, Li K, Fei-Fei L. Imagenet: a large-scale hierarchical image database. In 2009 IEEE conference on computer vision and pattern recognition (pp. 248-255), (2009, June).

12. Chaudhary Y, Mehta M, Sharma R, Gupta D, Khanna A, Rodrigues JJ. Efficient-CovidNet: deep learning based COVID-19 detection from chest $x$-ray images. In 2020 IEEE international conference on e-health networking, application \& services (HEALTHCOM) 2021 Mar 1 (pp. 1-6). IEEE.

13. Luz E, Silva P, Silva R, Silva L, Guimarães J, Miozzo G, Moreira G, Menotti D. Towards an effective and efficient deep learning model for COVID-19 patterns detection in X-ray images. Res Biomed Eng. 2021;20:1-4.

14. Pham TD. Classification of COVID-19 chest $X$-rays with deep learning: new models or fine tuning? Health Inf Sci Syst. 2021;9(1):1-1.

15. Saiz FA, Barandiaran I. COVID-19 detection in chest $X$-ray images using a deep learning approach. Int J Interact Multimed Artif Intell. 2020;6:4.

16. Rahimzadeh M, Attar A. A modified deep convolutional neural network for detecting COVID-19 and pneumonia from chest X-ray images based 
on the concatenation of Xception and ResNet50V2. Inf Med Unlocked. 2020;19:100360

17. Panwar H, Gupta PK, Siddiqui MK, Morales-Menendez R, Singh V. Application of deep learning for fast detection of COVID-19 in X-rays using nCOVnet. Chaos Solitons Fractals. 2020;138:109944.

18. Li J, Xi L, Wang X, et al. Radiology indispensable for tracking COVID-19. Diagn Interv Imaging. 2020;102(2):69-75.

19. Brunese L, Mercaldo F, Reginelli A, Santone A. Explainable deep learning for pulmonary disease and coronavirus COVID-19 detection from X-rays. Comput Methods Programs Biomed. 2020;196:105608.

20. Loey M, Smarandache F, Khalifa NEM. Within the lack of chest COVID-19 X-ray dataset: a novel detection model based on GAN and deep transfer learning. Symmetry. 2020;12(4):651.

21. OzturkT, Talo M, Yildirim EA, Baloglu UB, Yildirim O, Rajendra Acharya U. Automated detection of COVID- 19 cases using deep neural networks with X-ray images. Comput Biol Med. 2020;121:103792.

22. El Asnaoui K, Chawki Y. Using X-ray images and deep learning for automated detection of coronavirus disease. J Biomol Struct Dyn. 2020;38:3615-26.

23. Dey N, Rajinikanth V, Fong SJ, Kaiser MS, Mahmud M. Social group optimization-assisted Kapur's entropy and morphological segmentation for automated detection of COVID-19 infection from computed tomography images. Cogn Comput. 2020;12(5):1011-23.

24. Vaid S, Kalantar R, Bhandari M. Deep learning COVID-19 detection bias: accuracy through artificial intelligence. Int Orthopaed. 2020;44:1539-42.

25. Ucar F, Korkmaz D. COVIDiagnosis-Net: deep Bayes-SqueezeNet based diagnosis of the coronavirus disease 2019 (COVID-19) from X-ray images. Med Hypotheses. 2020;140:109761.

26. Togaçar M, Ergen B, Cömert Z. COVID-19 detection using deep learning models to exploit social mimic optimization and structured chest X-ray images using fuzzy color and stacking approaches. Comput Biol Med. 2020;121:103805.

27. Khan Al, Shah JL, Bhat MM. CoroNet: a deep neural network for detection and diagnosis of COVID-19 from chest X-ray images. Comput Methods Programs Biomed. 2020;196:105581.
28. Martinez F, Marínez F, Jacinto E. Performance evaluation of the NASNet convolutional network in the automatic identification of COVID-19. Int J Adv Sci Eng Inf Technol. 2020;10(2):662.

29. Waheed A, Goyal M, Gupta D, Khanna A, Al-Turjman F, Pinheiro PR. CovidGAN: data augmentation using auxiliary classifier GAN for improved COVID-19 detection. IEEE Access. 2020;8:91916-23.

30. Pereira RM, Bertolini D, Teixeira LO, Silla CN, Costa YMG. COVID-19 identification in chest X-ray images on flat and hierarchical classification scenarios. Comput Methods Programs Biomed. 2020;194:1055.

31. Apostolopoulos ID, Mpesiana TA. COVID-19: automatic detection from $X$-ray images utilizing transfer learning with convolutional neural networks. Phys Eng Sci Med. 2020;43(2):635-40.

32. Elaziz MA, Hosny KM, Salah A, Darwish MM, Lu S, Sahlol AT. New machine learning method for image-based diagnosis of COVID-19. PLoS ONE. 2020;15(6):e0235187.

33. Sethy PK, Behera SK, Ratha PK, Biswas P. Detection of coronavirus disease (COVID-19) based on deep features and support vector machine. Int J Math Eng Manag Sci. 2020;5(4):643-51.

34. Yi PH, Kim TK, Lin CT. Generalizability of deep learning tuberculosis classifier to COVID-19 chest radiographs: new tricks for an old algorithm? J Thoracic Imaging. 2020;35(4):102-4.

35. Das D, Santosh KC, Pal U. Truncated inception net: COVID-19 outbreak screening using chest X-rays. Phys Eng Sci Med. 2020;43(3):915-25.

36. COVID-19 Radiography Database. https://www.kaggle.com/tawsifurra hman/covid19-radiography-database. Accessed 3 May 2021.

37. Tan M, Le Q. Efficientnet: Rethinking model scaling for convolutional neural networks. International Conference on Machine Learning 2019 May 24 (pp. 6105-6114). PMLR.

38. Jiao Z, Choi JW, Halsey K, Tran TM, Hsieh B, Wang D, Eweje F, Wang R, Chang K, Wu J, Collins SA. Prognostication of patients with COVID-19 using artificial intelligence based on chest X-rays and clinical data: a retrospective study. The Lancet Digital Health. 2021;3(5):e286-94.

Publisher's Note Springer Nature remains neutral with regard to jurisdictional claims in published maps and institutional affiliations. 